\title{
Hydrothermal synthesis of anatase nanoleaves and size dependence of anatase-rutile transformation upon heating
}

\author{
T. V. Lisnycha ${ }^{1} \cdot$ S. A. Kirillov ${ }^{1,2}$ - A. V. Potapenko ${ }^{2} \cdot$ T. E. Terikovska ${ }^{1} \cdot$ \\ V. V. Kosilov ${ }^{2}$ O. A. Vyshnevskiy ${ }^{3}$
}

Received: 20 March 2015/Accepted: 9 December 2015/Published online: 22 January 2016

(c) The Author(s) 2016. This article is published with open access at Springerlink.com

\begin{abstract}
Amorphous $\mathrm{TiO}_{2}$ obtained by adding $\mathrm{TiCl}_{4}$ to an alkaline medium crystallizes slowly and upon 3 years ageing transforms to nanosized anatase containing an admixture of brookite. The hydrothermal treatment of this sample in solutions of lithium hydroxide leads to anatase nanoleaves, and the more concentrated $\mathrm{LiOH}$ solution, the greater the nanoleaves and the smaller their specific surface area. The thermal treatment of nanoleaves leads to the bulk rutile, and the greater the specific surface area of anatase nanoleaves, the lower the anatase-rutile transition temperature. This is in line with conclusions based on the thermodynamic stability of nanosized anatase over the bulk rutile.
\end{abstract}

Keywords Titanium oxide - Hydrothermal treatment . Anatase $\cdot$ Rutile $\cdot$ Phase transformation $\cdot$ Size dependence

\section{Introduction}

Titanium oxides are now widely investigated, first of all, as materials for energy conversion and storage, and advances in the field are reviewed thoroughly [1-7]. One of valuable applications of titania is its use as an anode in lithium-ion batteries, either itself or forming a part of lithium titanate,

S. A. Kirillov

kir@i.kiev.ua

1 Institute for Sorption and Problems of Endoecology, 13 Gen. Naumov St., Kiev 03164, Ukraine

2 Joint Department of Electrochemical Energy Systems, 38A Vernadsky Ave., Kiev 03142, Ukraine

3 Institute of Geochemistry, Mineralogy and Ore Formation, 34 Palladin Prosp., Kiev 03142, Ukraine
$\mathrm{Li}_{4} \mathrm{Ti}_{5} \mathrm{O}_{12}$, which is considered as the so-called zero-strain material demonstrating no volume change upon the insertion and deinsertion of lithium ions when the battery is charged and discharged [8].

Titanium oxide forms various polymorphs, and their stability markedly varies with the size of particles. Among titania polymorphs, viz., rutile, anatase, brookite, $\mathrm{TiO}_{2}-\mathrm{B}$, etc., anatase and, especially, $\mathrm{TiO}_{2}-\mathrm{B}$ demonstrate the best electrochemical performance [9]. Anatase and $\mathrm{Li}_{4} \mathrm{Ti}_{5} \mathrm{O}_{12}$ of electrochemical quality are usually obtained from organic derivatives of titanium. The synthesis of $\mathrm{TiO}_{2}-\mathrm{B}$ requires an autoclave recrystallization of a raw titanium oxide in a concentrated solution of $\mathrm{NaOH}$. Furthermore, the hydrothermal treatment in alkaline solutions is a wellknown mean of the synthesis of one-dimensional nanostructures of titania. It is suggested [10] that in hydrothermal solutions, sodium cations attack specific facets of the raw material and cause its exfoliation. The morphology of the reaction product (nanoribbons, nanotubes, nanofibers, nanowires) depends on the hydrothermal synthesis parameters, viz., temperature, pressure, and concentration of the solution.

The size dependence of mutual transformations between the $\mathrm{TiO}_{2}$ polymorphs is a topic of great attention of experimentalists. Preparation of materials often includes their thermal pretreatment, and this requires the knowledge regarding their phase state. Precise calorimetric measurements by Navrotsky and co-workers have shown [11, 12] that depending on the size, the thermodynamic stability of the $\mathrm{TiO}_{2}$ polymorphs at ambient temperatures is significantly different. For the smallest particles, the anatase structure is the most stable. Medium-sized particles prefer the structure of brookite. The greatest particles crystallize in the structure of rutile. Similar conclusions follow from solution studies where the anatase-to-rutile phase 
transformation and particle growth rates were observed to be size dependent and increase with decreasing initial particle size [13].

In our previous work [14], we have found that hydrolytic precipitation of $\mathrm{TiO}_{2}$ from titanium chloride can serve as a convenient alternative to the method of hydrolysis of organic derivatives of titanium. In particular, it has been noticed that depending on the conditions of the synthesis, titania samples having strictly different properties can be obtained. Neutralization of $\mathrm{TiCl}_{4}$ with the solution of an alkali metal hydroxide leads to an amorphous precipitate whose dehydration gives nanocrystalline anatase. Changing the order of mixing, i.e. adding $\mathrm{TiCl}_{4}$ to an alkaline medium leads to an amorphous precipitate of another type whose crystallization proceeds very slowly.

Continuing our efforts directed towards obtaining nanosized $\mathrm{TiO}_{2}$-based electrode materials by means of hydrolysis of titanium chloride $[14,15]$ we have subjected this hydrolytic titania to the hydrothermal treatment in concentrated solutions of $\mathrm{LiOH}$ instead of $\mathrm{NaOH}$. In this paper, we describe the products obtained from the aged precipitate synthesized by adding $\mathrm{TiCl}_{4}$ to $\mathrm{LiOH}$ and present the data regarding their transformations upon heating.

\section{Experimental}

A raw material for hydrothermal syntheses was obtained according the following procedure. The $\sim 5 \mathrm{M}$ solution of $\mathrm{TiCl}_{4}$ in hydrochloric acid was added dropwise to the solution of $\mathrm{LiOH}$ upon stirring. A precipitate was aged for $24 \mathrm{~h}$, filtered, rinsed with $0.1 \mathrm{M} \mathrm{HCl}$, washed with distilled water to the absence of chloride ions in the filtrate and then until pH 7 was reached, and air-dried (Sample 1). Ageing Sample 1 for 3 years in a closed vessel at ambient conditions gave Sample 2.

Hydrothermal syntheses were carried out at $200{ }^{\circ} \mathrm{C}$ and $18 \mathrm{~atm}$ upon stirring for $24 \mathrm{~h}$ in a Teflon-lined stainless steel autoclave. $0.27,0.43$ and $0.66 \mathrm{M}$ solutions of lithium hydroxide were mixed with Sample 2 so as to get the Ti:Li molar ratio of 1:1 (Sample 3), 1:1.6 (Sample 4) and 1:2.4 (Sample 5). Resulting materials were washed with distilled water until $\mathrm{pH} 7$ was reached and dried at $120{ }^{\circ} \mathrm{C}$. Their thermal treatment was made in a muffle furnace for $4 \mathrm{~h}$.

The morphology of samples was studied by powder X-ray diffraction (XRD) (DRON-4-07, LOMO, Russia, Cu $\mathrm{K} \alpha$ radiation, $\mathrm{Ni}$ filter) and scanning electron microscopy (SEM) (JSM 6100, JEOL, Japan). Surface areas and pore volumes were determined from nitrogen adsorption-desorption isotherms registered at $77 \mathrm{~K}$ on an ASAP 2000 instrument (Micromeritics, USA). Pore size distributions were calculated in terms of the Barrett-Joiner-Halenda (BJH) method.

\section{Results and discussion}

As follows from X-ray measurements (Fig. 1), the fresh precipitate (Sample 1) is amorphous. Weak, broad signals at $2 \theta=25.7$ and $37.5^{\circ}$ are characteristic reflections from (101) and (004) planes of anatase embryos, JCPDS \#211272. The XRD pattern of the aged precipitate (Sample 2) reveals the presence of anatase and brookite, JCPDS \#291360. The latter is discernible by reflections from (121), (032) and (132) planes at $2 \theta=30.9,46.2$ and $49.2^{\circ}$, respectively, which have no counterparts in anatase. The formation of an admixture of brookite in anatase in aged samples obtained from alkaline media has been already noticed in Ref. [16]. The crystallite size determined by means of the Scherrer method gives $R_{\text {cryst }}=3 \mathrm{~nm}$ (Table 1).

SEM and TEM micrographs of these materials have been discussed in Ref. [14] and are not shown here. At medium magnification (up to $\times 100,000$ ), they look like spheres of $20-30 \mathrm{~nm}$ diameter and are similar to that for Sample 3 subjected to the hydrothermal treatment, see below. At greater magnification in TEM, each sphere appears consisting of smaller spherical particles of 6-8 nm in diameter.

Nitrogen adsorption-desorption isotherms and pore size distributions for fresh and aged precipitates are shown in Fig. 2; porosimetric data are summarized in Table 1. Ageing leads to a decrease in specific surface areas $S_{\mathrm{sp}}$. and

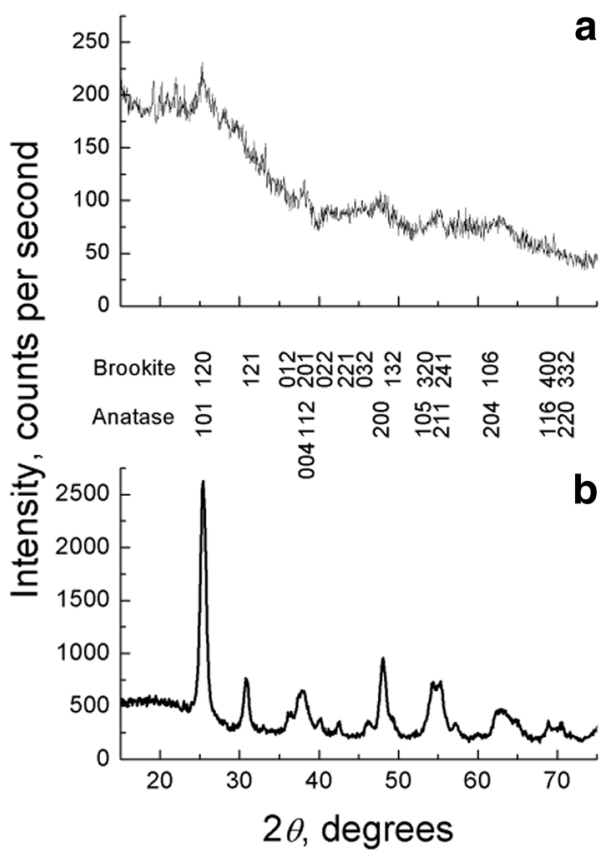

Fig. 1 X-ray diffraction patterns of fresh (a) and aged $\mathrm{TiO}_{2}$ (b) and Miller indices for reflections of anatase (JCPDS \#21-1272) and brookite (JCPDS \#29-1360) 
Table 1 Parameters of fresh, aged and hydrothermally treated $\mathrm{TiO}_{2}$

\begin{tabular}{|c|c|c|c|c|c|c|c|c|c|c|}
\hline \multirow[t]{2}{*}{ Sample } & \multirow[t]{2}{*}{ No. } & \multirow[t]{2}{*}{ Ti:Li ratio } & \multirow[t]{2}{*}{$S_{\mathrm{sp}} \cdot\left(\mathrm{m}^{2} \mathrm{~g}^{-1}\right)$} & \multirow[t]{2}{*}{$V_{\text {pore }}\left(\mathrm{cm}^{3} \mathrm{~g}^{-1}\right)$} & \multicolumn{2}{|c|}{$R_{\text {pore }}(\mathrm{nm})$} & \multicolumn{2}{|c|}{$R_{\text {part }}(\mathrm{nm})$} & \multirow[t]{2}{*}{$R_{\text {cryst }}(\mathrm{nm})$} & \multirow[t]{2}{*}{$\rho$} \\
\hline & & & & & BJH & GGP & SEM & GGP & & \\
\hline Fresh & 1 & - & 430 & 0.349 & 1.8 & 1.6 & $3-4$ & 1.64 & - & 0.50 \\
\hline Aged & 2 & - & 217 & 0.586 & 4 & 5.4 & $3-4$ & 3.25 & 3 & 0.38 \\
\hline Hydrothermal & 3 & $1: 1$ & 83 & 0.248 & 4 & 6.0 & $<20$ & 8.42 & 12 & 0.59 \\
\hline Hydrothermal & 4 & $1: 1.6$ & 57 & 0.145 & 4 & 5.1 & $<20$ & 7.99 & 12 & 0.71 \\
\hline Hydrothermal & 5 & $1: 2.4$ & 38 & 0.088 & 4 & $<5.3$ & - & $<9.17$ & 12 & 0.78 \\
\hline
\end{tabular}

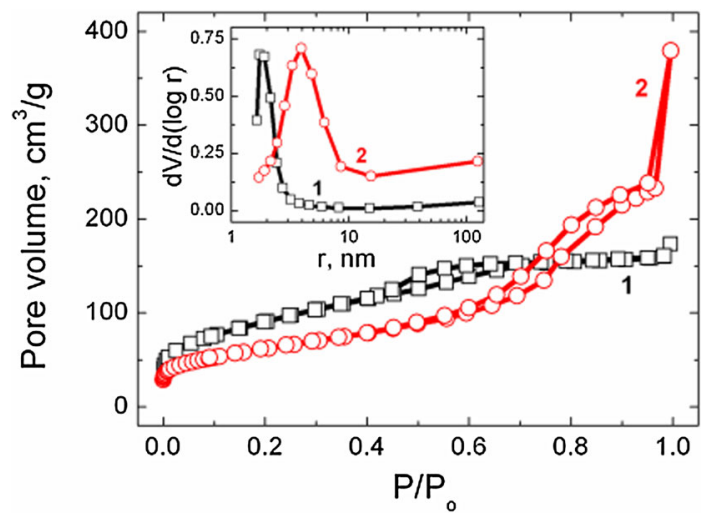

Fig. 2 Nitrogen adsorption-desorption isotherms and pore size distributions (inset) for fresh (1) and aged (2) samples. Curves are numbered according to Table 1

an increase in pore volumes $V_{\text {pore }}$ and pore radii $R_{\text {pore }}$. Such a trend can be understood in terms of the gapped gapless packing (GGP) model [17]. This model considers the porous substance as an ensemble of rigid spherical particles and enables one to calculate the values of $R_{\text {pore }}$, the particle radii $R_{\text {part }}$, and the packing fraction $\rho$ based on the data on $S_{\mathrm{sp}}$. and $V_{\text {pore }}$. The packing fraction lies in the following limits, $0.78>\rho>0.39$; the upper limit corresponds to a densely packed system, whereas below the lower limit the particles cannot be kept together and get scattered. The measured and calculated $R_{\text {pore }}$ values are close (Table 1 ). An increase in $R_{\text {part }}$ accompanied by a decrease in the packing fraction signifies that ageing leads to the growth of the particles and weakens attraction between them.

The hydrothermal treatment significantly changes the morphology of the samples studied. SEM micrographs demonstrate a gradual transformation of spherical particles to nanoleaves (Fig. 3). For Sample 3 exposed to heating in the least concentrated solution $(0.27 \mathrm{M} \mathrm{LiOH})$, sporadic nanoleaves (in circles) can be noticed on the surface of the bulk $\mathrm{TiO}_{2}$. SEM data give an impression that nanoleaves are the products of the surface coalescence of spherical particles constituting the raw material. Groups of transparent (i.e. very thin) nanoleaves are discernible in the case of Sample 4 processed in the medium concentrated solution
(0.43 M LiOH). The transformation completes in the most concentrated solution (0.66 M LiOH), Sample 5 .

Nitrogen adsorption-desorption isotherms and pore size distributions for hydrothermally treated samples are given in Fig. 4; porosimetric data are listed in Table 1. The very view of the isotherms is close to that of the aged sample. $S_{\text {sp. }}$ and $V_{\text {pore }}$ gradually decrease if compared with those for the raw material. $R_{\text {pore }}$ derived by means of the BJH procedure appears the same and equal to that of the aged sample. These remain constant irrespective of the concentration of $\mathrm{LiOH}$ in the solution and are too small to be ascribed to interstices between particles. Probably, $R_{\text {pore }}$ characterizes the microporosity of nanoleaves. Noticeably, the $R_{\text {pore }}$ values determined by means of the BJH procedure and using the GGP model are close.

The X-ray diffraction data (Fig. 5) signify that the hydrothermal treatment gradually decreases the amount of brookite in the samples. One may suggest that nanoleaves consist of pure anatase, and the signature of brookite in XRD patterns corresponds to the non-recrystallized raw material. The crystallite size determined by means of the Scherrer method using the reflections from (101) and (004) planes of anatase gives $R_{\text {cryst }}$ of ca. $12 \mathrm{~nm}$ (Table 1). This is in good agreement with calculations of $R_{\text {part }}$ performed within the GGP model. It is tempting to speculate than this $R_{\text {part }}$ corresponds to the thickness of the nanoleaves, which may be considered as aggregates of single nanocrystals of $R_{\text {cryst }}$ size.

Having three nanodimensional anatase samples of different particle size and morphology, it is of great interest to study their transformation upon heating. The data obtained by Navrotsky et al. [11, 12], Fig. 6, give a guideline for understanding this process where two opportunities may be foreseen. If the $\mathrm{TiO}_{2}$ particles are of equal size, rutile is thermodynamically stable within $0<S_{\mathrm{sp} .}<7 \mathrm{~m}^{2} \mathrm{~g}^{-1}$, whereas both brookite and anatase are metastable against it; brookite becomes thermodynamically stable within $7<S_{\mathrm{sp}} .<46 \mathrm{~m}^{2} \mathrm{~g}^{-1}$, and both rutile and anatase are metastable against it; finally, anatase remains the only thermodynamically stable $\mathrm{TiO}_{2}$ phase upon subdivision to $S_{\mathrm{sp} .}>46 \mathrm{~m}^{2} \mathrm{~g}^{-1}$, and both rutile and brookite are metastable against it [11]. In this case, one may expect that should the particle size not vary upon heating, the anatase 


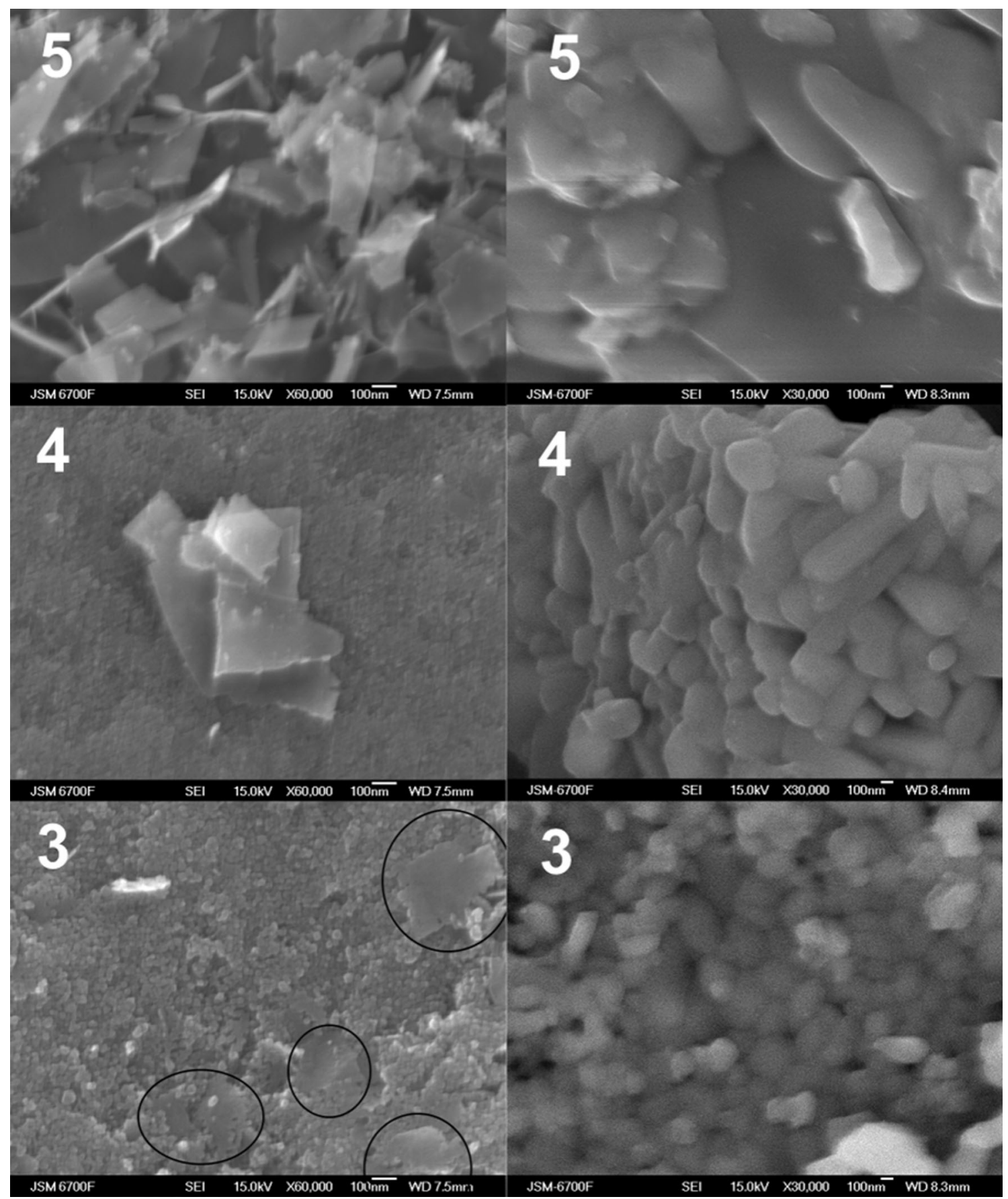

Fig. 3 SEM micrographs of samples upon hydrothermal treatment $(l e f t)$ and upon heating at $1000{ }^{\circ} \mathrm{C}$. Sample numbering according to Table 1

structure of the samples studied would be preserved. On the other hand, both anatase and brookite are metastable against the bulk rutile, and the smaller the particle, the more heat is evolved upon the anatase-bulk rutile transformation [12]. In this case, one may anticipate that should aggregation occur upon heating, rutile would be formed as the most thermodynamically stable phase, and the greater the $S_{\mathrm{sp}}$. value, i.e. the smaller the anatase particles, the less heat should be absorbed for their transformation to rutile and the lower the transformation temperature that would be observed. Besides these thermodynamic factors, differences in anatase-rutile transformation temperature may arise by kinetic or catalytic reasons.

The X-ray diffraction data for heated samples are collected in Fig. 7. It is well seen that the most dispersed 


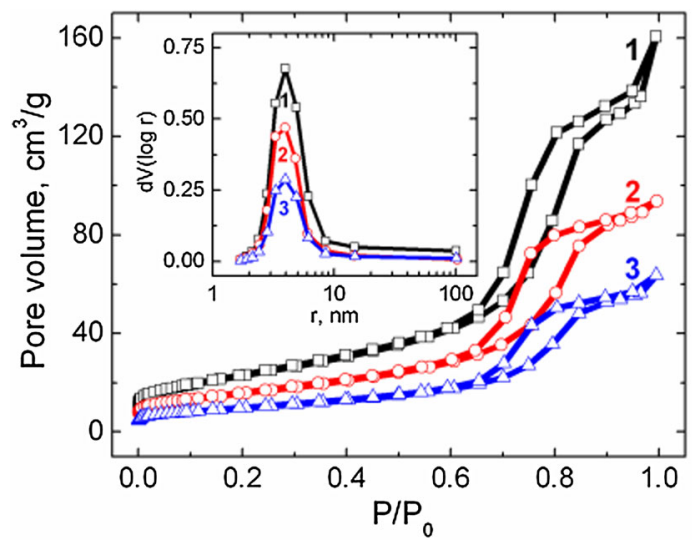

Fig. 4 Nitrogen adsorption-desorption isotherms and pore size distributions (inset) hydrothermally treated samples. Curves are numbered according to Table 1

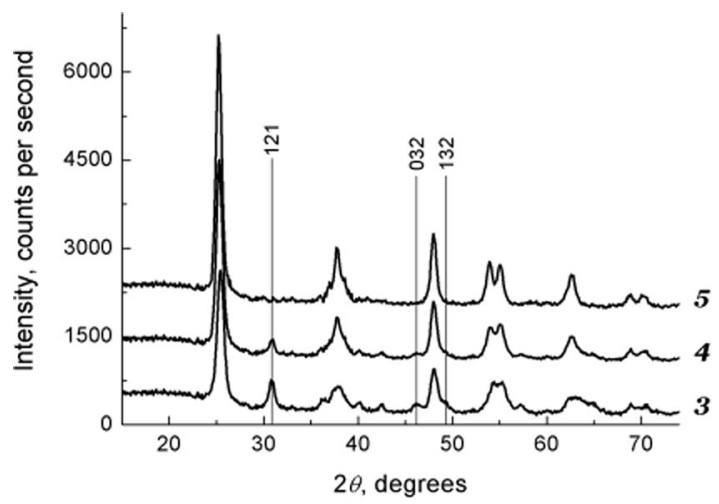

Fig. 5 X-ray diffraction patterns of hydrothermally treated samples. Specified are distinctive reflections from (121), (032) and (132) planes of brookite at $2 \theta=46.1$ and $49.2^{\circ}$, JCPDS \#29-1360

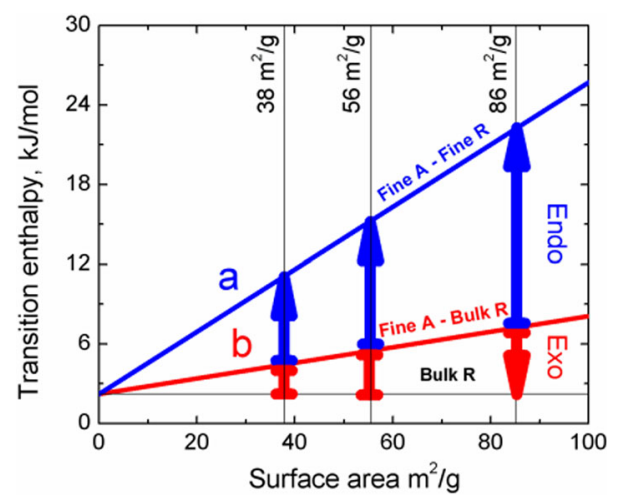

Fig. 6 Enthalpy of anatase vs. rutile. a Anatase and rutile particles of the same size. b Anatase of various particle size vs. bulk rutile

Sample 3 demonstrates the transition to rutile at $\sim 700{ }^{\circ} \mathrm{C}$; Sample 4 of intermediate particle size transforms to rutile at $\sim 800{ }^{\circ} \mathrm{C}$; the least dispersed Sample 5 appears the most heat resistive and turns into rutile at $\sim 1000{ }^{\circ} \mathrm{C}$. It should
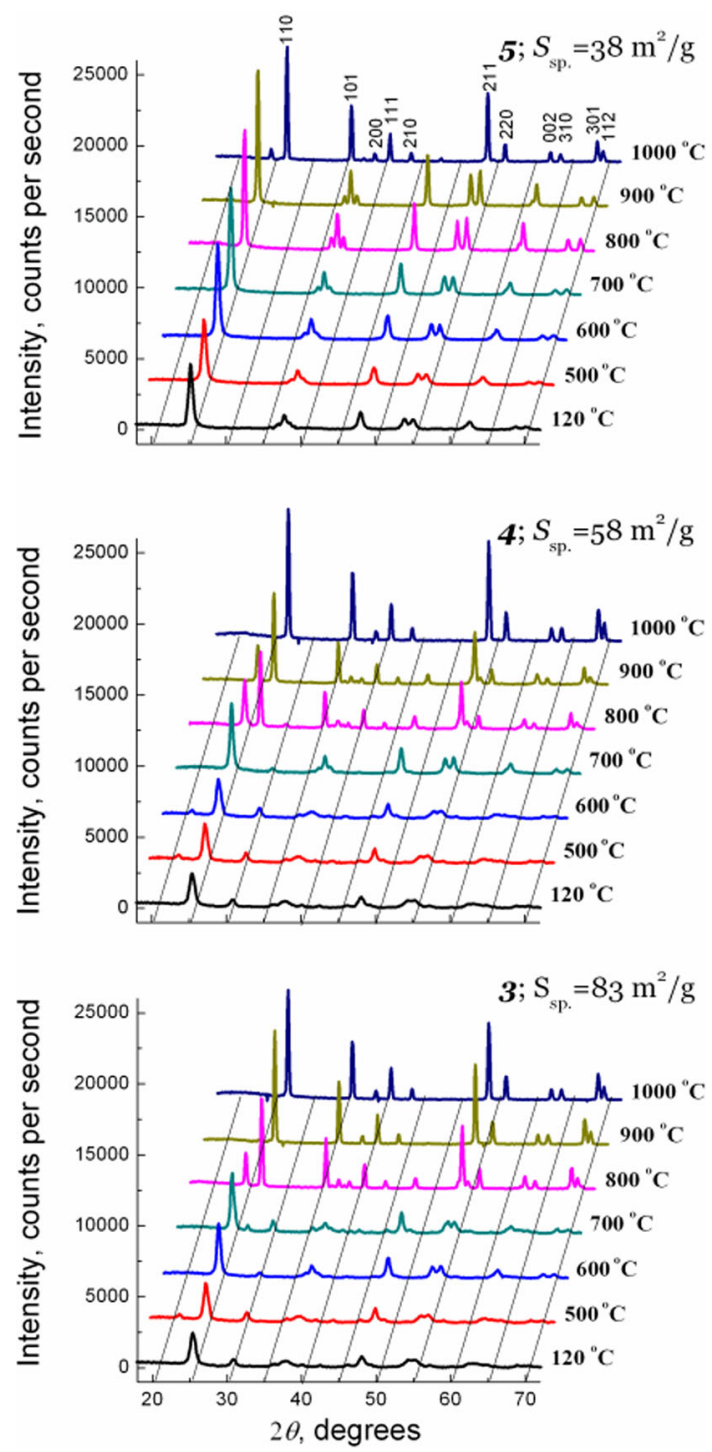

Fig. 7 X-ray diffraction patterns of hydrothermally treated samples annealed at various temperatures. Miller indices for rutile (JCPDS \#21-1276) are shown on the top; for Miller indices for anatase and brookite see Fig. 1

be mentioned that in the latter case, anatase-rutile transformation is not fully complete, and remnants of anatase are evident in Fig. 7 as reflections from (101) and (200) planes of anatase at $2 \theta=25.4$ and $48.1^{\circ}$.

Calculations reassure that upon heating, the crystallite size determined from these reflections of anatase and from reflections from (110) and (101) planes of rutile at $2 \theta=27.4$ and $36.0^{\circ}$, JCPDS \#21-1276, within the limit of uncertainty is the same in all samples (Fig. 8) and hence has nothing to do with the differences in the transition temperature. Possible catalytic influence of brookite can also be disregarded since the careful inspection of the XRD patterns evidences that brookite disappears turning to anatase before the anatase-rutile transformation. This 


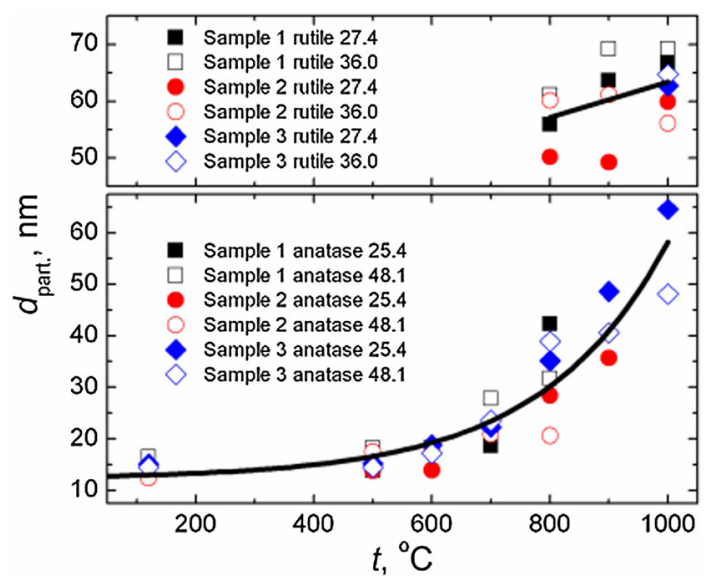

Fig. 8 Crystallite size in various samples upon heating. Solid lines are the mean crystallite sizes averaged over Samples 3, 4 and 5

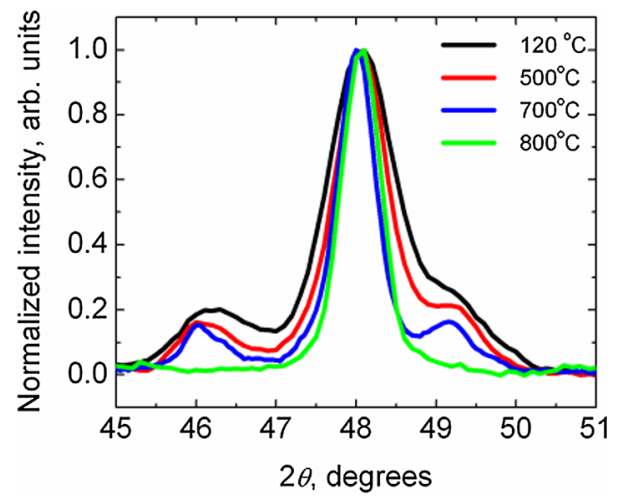

Fig. 9 X-ray diffraction patterns of hydrothermally treated samples annealed at various temperatures in the region of the (032) and (132) reflections of brookite. The strong line at $2 \theta=48.0$ corresponds to the (200) reflection of anatase

conclusion has been drawn from the analysis of the characteristic reflections from (032) and (132) planes of brookite (Fig. 9).

SEM micrographs signify that the morphology of the thermally treated samples differs from that of parent samples (Fig. 3). Heating leads to aggregation of particles. The higher the concentration of the alkali and the more the anatase nanoleaves are present in the hydrothermally treated samples, the greater are the rutile particles obtained upon heating. Nitrogen adsorption-desorption curves demonstrate no hysteresis loops and hence no porosity; the BET method gives $S_{\mathrm{sp}} \sim 1.2 \mathrm{~m}^{2} \mathrm{~g}^{-1}$. Using a formula connecting the particle size (in $\mathrm{nm}$ ) and $S_{\mathrm{sp}}$. for spherical loosely packed particles, $d_{\mathrm{part}}=6 \times 10^{-6} /\left(S_{\mathrm{sp}} \cdot \rho\right)$, where $\rho$ is the density one obtains $d_{\text {part }} \approx 1 \mu \mathrm{m}$. This means that heating hydrothermally treated samples leads to the bulk rutile. Therefore, the differences in the anataserutile transition temperatures should be explained in terms of exothermic transition of nanosized anatase-bulk rutile.

\section{Conclusions}

In this paper, we inform that amorphous $\mathrm{TiO}_{2}$ obtained by adding $\mathrm{TiCl}_{4}$ to an alkaline medium crystallizes slowly and upon 3 years ageing transforms to nanosized anatase containing an admixture of brookite. The hydrothermal treatment of this sample in $0.27,0.43$ and $0.66 \mathrm{M}$ solutions of lithium hydroxide leads to anatase nanoleaves, and the more concentrated $\mathrm{LiOH}$ solution, the greater the nanoleaves and the smaller their specific surface area decreasing from 83 to $38 \mathrm{~m}^{2} \mathrm{~g}^{-1}$. The thermal treatment of nanoleaves leads to the bulk rutile and the most dispersed sample demonstrates the transition to rutile at $\sim 700{ }^{\circ} \mathrm{C}$, whereas the least dispersed one turns into rutile at $\sim 1000{ }^{\circ} \mathrm{C}$. This observation is in line with conclusions based on the thermodynamic stability of nanosized anatase over bulk rutile.

\section{Compliance with ethical standards}

Conflict of interest The authors declare no competing interests.

Authors' contributions TVL and TET carried out hydrothermal experiments and prepared the first version of the manuscript. VVK participated in hydrothermal studies. AVP determined the crystallite size from XRD data. OAV registered and described SEM data. SAK wrote the final manuscript. All authors read and approved the final manuscript.

Open Access This article is distributed under the terms of the Creative Commons Attribution 4.0 International License (http://creative commons.org/licenses/by/4.0/), which permits unrestricted use, distribution, and reproduction in any medium, provided you give appropriate credit to the original author(s) and the source, provide a link to the Creative Commons license, and indicate if changes were made.

\section{References}

1. Chen, X., Mao, S.S.: Titanium dioxide nanomaterials: synthesis, properties, modifications, and applications. Chem. Rev. 107, 2891-2959 (2007)

2. Zhou, W., Liu, H., Boughton, R.I., Du, G., Lin, J., Wang, J., Liu, D.: One-dimensional single-crystalline Ti-O based nanostructures: properties, synthesis, modifications and applications. J. Mater. Chem. 20, 5993-6008 (2010)

3. Chen, X., Selloni, A.: Introduction: titanium dioxide $\left(\mathrm{TiO}_{2}\right)$ nanomaterials. Chem. Rev. 114, 9281-9282 (2014)

4. Cargnello, M., Gordon, T.R., Murray, C.B.: Solution-phase synthesis of titanium dioxide nanoparticles and nanocrystals. Chem. Rev. 114, 9319-9345 (2014)

5. Wang, X., Li, Z., Shi, J., Yu, Y.: One-dimensional titanium dioxide nanomaterials: nanowires, nanorods, and nanobelts. Chem. Rev. 114, 9346-9384 (2014)

6. Wang, L., Sasaki, T.: Titanium oxide nanosheets: graphene analogues with versatile functionalities. Chem. Rev. 114, 9455-9486 (2014)

7. Bourikas, K., Kordulis, C., Lycourghiotis, A.: Titanium dioxide (anatase and rutile): surface chemistry, liquid-solid interface chemistry, and scientific synthesis of supported catalysts. Chem. Rev. 114, 9754-9823 (2014) 
8. Ohzuku, T., Ueda, A., Yamamoto, N.: Zero-strain insertion material of $\mathrm{Li}\left[\mathrm{Li}_{1 / 3} \mathrm{Ti}_{5 / 3}\right] \mathrm{O}_{4}$ for rechargeable lithium cells. J. Electrochem. Soc. 142, 1431-1435 (1995)

9. Yang, Z., Choi, D., Kerisit, S., Rosso, K.M., Wang, D., Zhang, J., Graff, G., Liu, J.: Nanostructures and lithium electrochemical reactivity of lithium titanites and titanium oxides: a review. J. Power Sources 192, 588-598 (2009)

10. Wu, D., Liu, J., Zhao, X., Li, A., Chen, Y., Ming, N.: Sequence of events for the formation of titanate nanotubes, nanofibers, nanowires, and nanobelts. Chem. Mater. 18, 547-553 (2006)

11. Ranade, M.R., Navrotsky, A., Zhang, H.Z., Banfield, J.F., Elder, S.H., Zaban, A., Borse, P.H., Kulkarni, S.K., Doran, G.S., Whitfield, H.J.: Energetics of nanocrystalline $\mathrm{TiO}_{2}$. Proc. Natl. Acad. Sci. 99, 6476-6481 (2002)

12. Levchenko, A.A., Li, G., Boerio-Goates, J., Woodfield, B.F., Navrotsky, A.: $\mathrm{TiO}_{2}$ stability landscape: polymorphism, surface energy, and bound water energetics. Chem. Mater. 18, 6324-6332 (2006)
13. Sabyrov, K., Burrows, N.D.: Lee Penn R.: size-dependent anatase to rutile phase transformation and particle growth. Chem. Mater. 25, 1408-1415 (2013)

14. Kirillov, S.A., Lisnycha, T.V., Chernukhin, S.I.: Precipitated nanosized titanium dioxide for electrochemical applications. J. Power Sources 196, 2221-2226 (2011)

15. Lesnichaya, T.V., Terikovskaya, T.E., Kosilov, V.V., Kirillov, S.A.: Synthesis, morphology and electrochemical properties of $\mathrm{TiO}_{2}$ microspheres. Chem. Phys. Tech. Surf. 6, 190-195 (2015)

16. Jalava, J.-P., Heikkilä, L., Hovi, O., Laiho, R., Hakanen, A., Härmä, H.: Structural investigation of hydrous $\mathrm{TiO}_{2}$ precipitates and their aging products by X-ray diffraction, atomic force microscopy, and transmission electron microscopy. Ind. Eng. Chem. Res. 37, 1317-1323 (1998)

17. Kirillov, S.A.: Surface area and pore volume of a system of particles as a function of their size and packing. Microporous Mesoporous Mater. 122, 234-239 (2009) 